\title{
Cavitating flow control through continuous tangential mass injection on a 2D hydrofoil at a small attack angle
}

\author{
Mikhail V. Timoshevskiy ${ }^{1,2}$, Ivan I. Zapryagaev ${ }^{1,2}$, Konstantin S. Pervunin ${ }^{1,2, a}$ and Dmitriy M. \\ Markovich ${ }^{1,2,3}$ \\ ${ }^{1}$ Kutateladze Institute of Thermophysics, Siberian Branch of the Russian Academy of Sciences, 1, Lavrentyev Ave., \\ Novosibirsk, 630090, Russia \\ ${ }^{2}$ Department of Physics, Novosibirsk State University, 2, Pirogov Str., Novosibirsk, 630090, Russia \\ ${ }^{3}$ Institute of Power Engineering, Tomsk Polytechnic University, 30, Lenin Ave., Tomsk, 634050, Russia
}

\begin{abstract}
We studied cavitating flow over the suction side of a symmetric 2D foil - a scaleddown model of high-pressure hydroturbine guide vanes $(\mathrm{GV})$ - in different cavitation regimes at the attack angle of $3^{\circ}$. High-speed imaging was used to analyze spatial patterns and time dynamics of the gas-vapour cavities. A hydroacoustic pressure transducer was employed to register time-spectra of pressure fluctuations nearby the hydrofoil. A PIV technique was applied to measure the velocity fields and its fluctuations. The active flow control was implemented by means of a continuous liquid supply with different flow rates through a slot channel located in the GV surface. It was found that the active mass injection does not influence the primary flow upstream of the slot channel position. For the cavitation-free and cavitation inception cases, the injection was shown to make the turbulent wake past the GV section more intense. However, at the developed cavitation regimes the active flow management made it possible to reduce substantially the amplitude or even totally suppress the periodic cavity length oscillations and pressure pulsations associated with them.
\end{abstract}

\section{Introduction}

Unsteady cavitation phenomena occurring within the ducts of real hydropower systems are known to be one of the main sources of instabilities that cause significant vibrations, overloads and, consequently, fatigue damages. These might lead to unstable operating conditions, reduced efficiency and safety problems. Presently, two main classes of partial cavity instabilities are distinguished [1]: intrinsic and system. In order to hinder the development of the unsteadiness a number of methods are applied to manipulate the flow dynamics. Probably, the most simple in implementation and effective of them is continuous tangential mass injection along a test body surface to introduce an additional momentum to overcome the adverse pressure gradient [2].

The paper aims at the experimental study of partial cavity flows around a scaled-down model of guide vanes $(\mathrm{GV})$ of a high-pressure turbine at a small attack angle, different cavitation numbers and control of the cavitation by means of continuous mass injection through a spanwise slot channel in the foil surface. We report here on high-speed visualization of spatial structure and dynamics of attached

\footnotetext{
${ }^{\text {a }}$ Corresponding author: pervunin@itp.nsc.ru
} 
and cloud cavities and their integral characteristics, a comparison of the frequency spectra of pressure pulsations near the profile made by a hydrophone and PIV measurements of the spatial distributions of the mean velocity and its turbulent fluctuations around the cavitating GV model.

\section{Test foil and experiment description}

The test foil of $C=100 \mathrm{~mm}$ chord length was a scaled-down model of a Francis turbine guide vane $(\mathrm{GV})$, which is a two-dimensional body, with a blunt trailing edge of $1.31 \mathrm{~mm}$ height (Fig. 1). It was made of brass with the mean surface roughness of $1.5 \mu \mathrm{m}$. The maximum width of $\mathrm{GV}$ is $H_{\max }=$ $0.2206 C$ measured at the distance of $x_{\max }=0.4396 C$ from its leading edge. The aspect ratio (the ratio of the foil span to its chord) was $\mathrm{AR}=0.8$ that is very close to the one in some practical full-scale conditions.

In order to provide flow control, the hydrofoil was manufactured with a spanwise slot channel in its surface (Fig. 1) through which the liquid was supplied from a bypass circuit directly to the test section along the GV surface. The slot nozzle exit of $0.6 \mathrm{~mm}$ height and $70 \mathrm{~mm}$ width was located at the distance of $0.6 \mathrm{C}$ from the foil leading edge so that the liquid was injected across the whole foil span, except for the end caps of $5 \mathrm{~mm}$ width. The nozzle chamber inside the GV profile had a helical geometry with a contraction towards the exit (Fig. 1a). The maximum exit velocity of the slot wall jet was $U_{\text {in }}=10.6 \mathrm{~m} / \mathrm{s}$. The measurement uncertainty of the injection flow rate did not exceed $2 \%$.
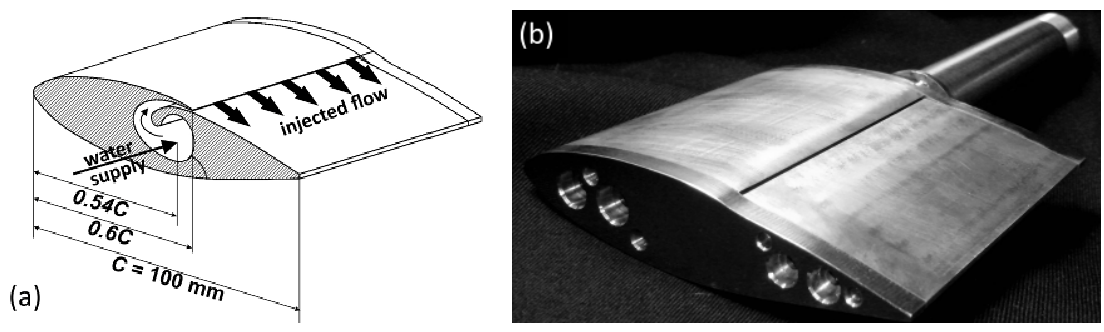

Figure 1. (a) A 3D scheme and (b) photograph of the fabricated GV profile.

The experiments were carried out in the Cavitation tunnel in Kutateladze Institute of Thermophysics SB RAS. Its description as well as the details on experimental conditions and applied measurement techniques can be found in [3]. The foil attack angle $\alpha$ was $3^{\circ}$. Pressure measurements were carried out close to the test foil by means of a hydroacoustic pressure transducer - a hydrophone B\&K Type 8103 that was flush-mounted into the sidewall of the test section. It was vertically located at the centre of the test section and $80 \mathrm{~mm}$ shifted behind the GV trailing edge.

\section{Results}

\subsection{Visualization and pressure measurements}

Cavitation is initiated right behind the leading edge on the suction side of the GV profile in form of travelling bubbles (Fig. 2-1.a). At $\sigma=0.95$, initially transitional cavity that is characterized by relatively small clouds (compared to cloud cavitation regimes) shedding from the cavity closure region is transformed to a typical travelling bubble cavitation as soon as the injection through the slot is started (Fig. 2-1.b). This leads to a decrease in the dimensions of the cavitation area on the GV surface. However, pressure fluctuations for both cases are almost identical and reveal no effect of the injection (Fig. 2-1.c).

This influence detected visually is probably linked with the existence of a shear layer past the backward facing step produced by the slot and its wall, which leads to an abrupt reduction of the shear stresses once the injection begins. Weakened shear stresses cannot anymore produce strong spanwise 
vortices (like in the case without injection) in the centres of which an intense evaporation occurs and, thus, cavitation is suppressed.

When the cavitation number is slightly decreased to 0.89 , the partial cavity on the GV section becomes unsteady and starts to oscillate periodically in the unforced flow case (Fig. 2-2.a). This is also proved by the pressure measurements. The main maximum that is clearly seen in Fig. 2-2.c at St $=f C / U_{0}=0.21$ corresponds to the frequency of the attached cavity pulsations. At this regime, the liquid injection results in stabilization of the attached cavity dynamics (Fig. 2-2.b), changing the partial cavity regime to transitional one like that shown in Fig. 2-1.a.

At the same time, the peak in the pressure fluctuations spectrum at $\mathrm{St}=0.21$ vanishes completely (Fig. 2-2.c). When the cavitation number is further reduced to $\sigma=0.84$, the active mass injection does not already change the cavity unsteady behaviour (Figs. 2-3.a and 2-3.b) but the amplitude of the pressure pulsations diminishes about three times (cf. the peak heights in Fig. 2-3.c).
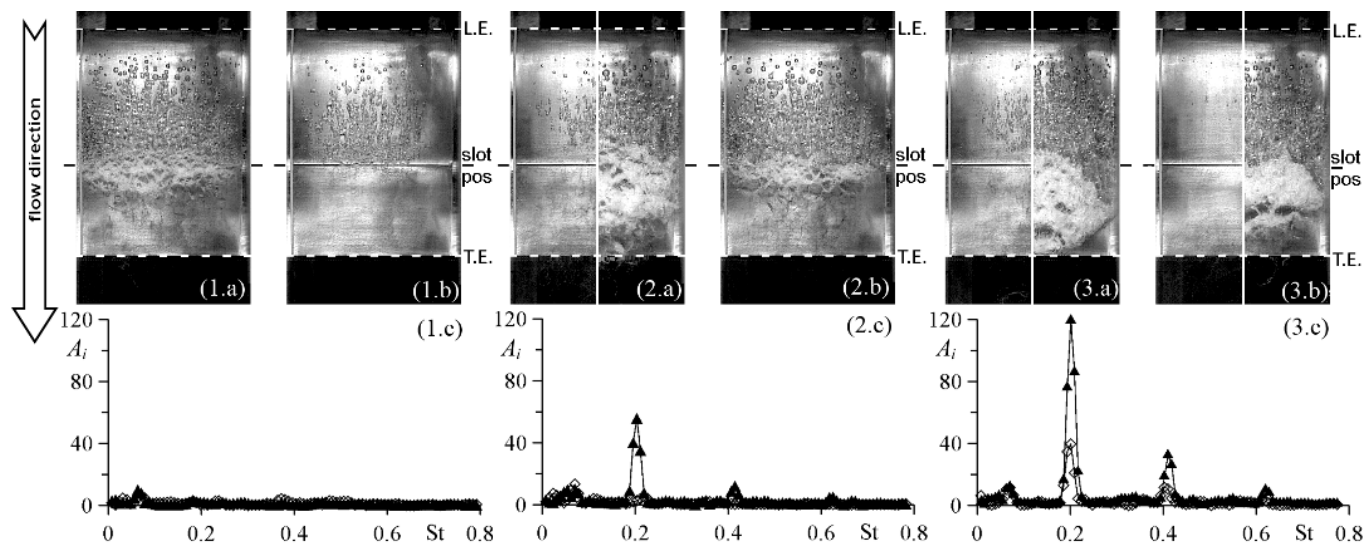

Figure 2. Instantaneous images of partial cavities (top view) on the suction side of the GV model at $\alpha=3^{\circ}$ (a) without and (b) with mass injection through the slot channel and (c) comparison of the frequency spectra of pressure pulsations, where $A_{i}$ denotes fast-Fourier-transform coefficients $(\diamond-$ with injection, $\mathbf{\Delta}-$ w/o injection), when: (1) $\sigma=0.95, U_{i n} / U_{0}=0.79$ (transitional cavitation/travelling bubbles), (2) $\sigma=0.89, U_{i n} / U_{0}=0.74$ (unsteady cavity/ transitional cavitation) and (3) $\sigma=0.84, U_{i n} / U_{0}=0.8$ (unsteady cavitation in both cases). For unsteady regimes, half-images indicating the phases of the attached cavity evolution when it is of (left) the shortest (just before its detachment) and (right) maximal lengths are shown together.

\subsection{Velocity distributions}

Fig. 3 display selected profiles of the streamwise component of the time-averaged velocity (plotted as the normalized velocity defect, $\left.\left(U-U_{0}\right) / U_{0}\right)$ and the rms of the streamwise component of its turbulent fluctuations $\tilde{u}$ at several locations along the GV model. The reference point (the origin of coordinate axes) coincides with the leading edge of the GV section in the measurement plane at $\alpha=0^{\circ}$.

As seen, the injection does not affect the profiles of the mean and fluctuating velocities upstream of the slot channel position at all (cf. the profiles at sections $x / C=0.1$ and 0.4 in Fig. 3). Moreover, the injection flow rate corresponding to the range of the injection velocities from $U_{i n} / U_{0}=0$ to 0.76 was found not to practically influence the distributions of these characteristics (not shown in the images) so that the global difference is only between the unforced and forced flow conditions. Thus, below only these two cases are considered, without taking into account the values of $U_{i n} / U_{0}$.

Besides the aforementioned modification of the cavitation pattern from transitional cavitation to travelling bubbles (Figs. 3-1.a and 3-2.a), the mass injection also alters noticeably the distributions of the mean velocity and turbulence intensity within the boundary layer, decreasing the boundary layer thickness by half. Thus, at these regimes the active control system allows a favourable and efficient flow manipulation.

When transition to unsteady regimes occurs (Figs. 3-1.b and 3-1.c), the mass injection does not, however, influence the transversal size of the boundary layer but somewhat reduces the mean flow 
velocity inside it. In addition, under the forced conditions, the turbulent fluctuations are in general lower (typically by roughly 30\%) in comparison with the unforced flow conditions (Figs. 3-2.b and 32.c).
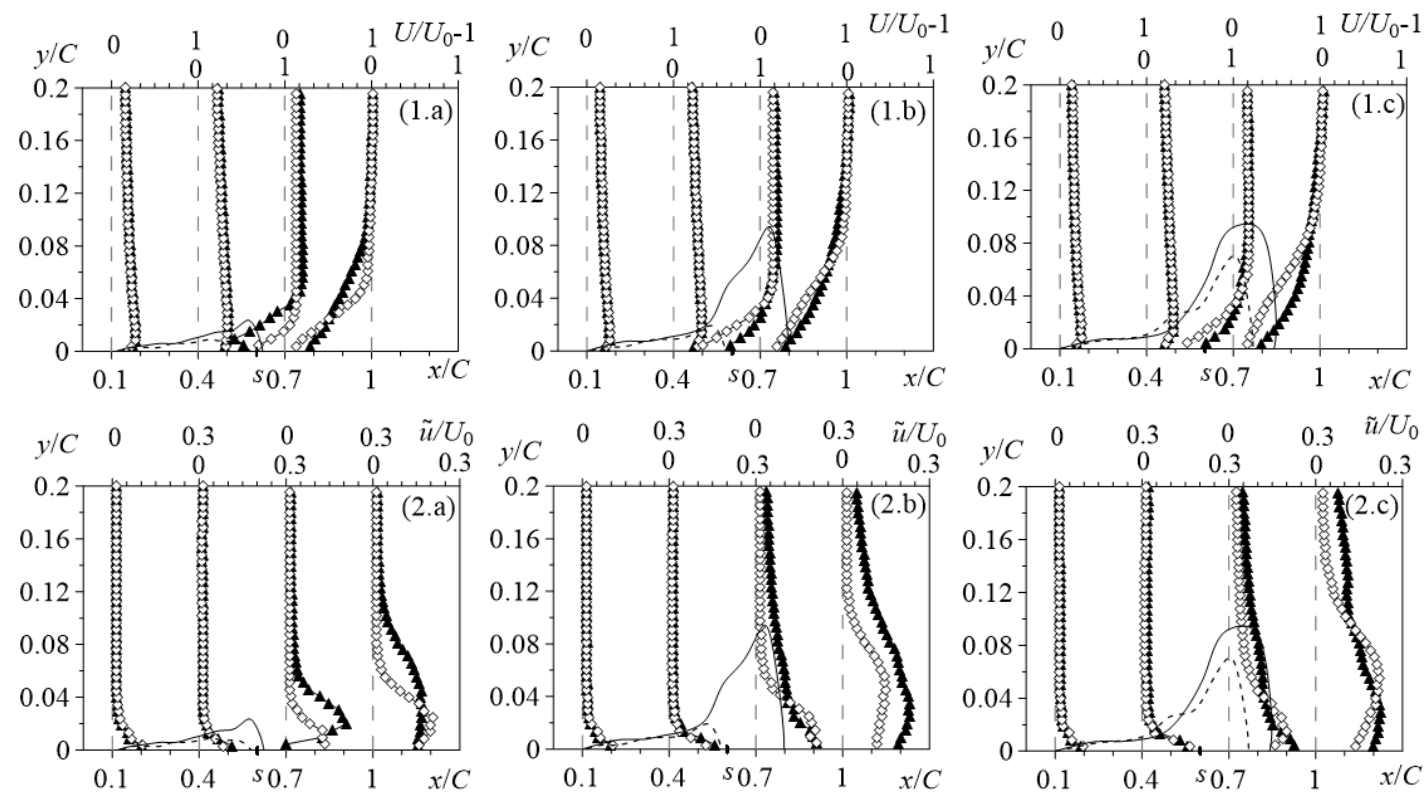

Figure 3. Downstream evolution of the streamwise component of the (1) mean velocity and (2) turbulence intensity (rms values) over the suction side of the GV model for (a) transitional cavitation/traveling bubbles at $\sigma=$ $0.95, U_{i n} / U_{0}=0.66$; (b) unsteady cavity/transitional cavitation at $\sigma=0.89, U_{i n} / U_{0}=0.74$ and (c) unsteady cavitation in both cases at $\sigma=0.84, U_{\text {in }} / U_{0}=0.76 . \alpha=3^{\circ} . \Delta-$ w/o injection, $\diamond-$ with injection. Solid and dashed lines represent interfaces (extracted from visual data) of the attached cavities of maximum size on the GV section for the unforced and forced flow conditions. $s$ denotes the position of the slot channel in the foil surface. The flow direction is from the left.

\section{Conclusions}

The continuous liquid supply through a slot channel in the GV section surface allows a favourable and efficient flow manipulation, especially at the regimes with developed cavitation. In particular, the flow management makes it possible to reduce substantially the amplitude or totally suppress the periodic cavity length oscillations and pressure pulsations associated with them. However, the physics of the regarded effects remains unclear, which, therefore, requires supplementary experiments for a broader range of the governing parameters.

Acknowledgments. The research was funded by a grant from the Russian Scientific Fund (Project No. 16-19-00138) through IT SB RAS.

\section{References}

1. M. Callenaere, J.-P. Franc, J.-M. Michel and M. Riondet, J. Fluid Mech. 444, 223-256 (2001)

2. P.G. Kozhukharov, V.H. Hadjimikhalev, V.I. Mikuta, L.I. Maltzev, ASME Fluids Eng. Div. (Int. Symp. Jets and Cavities, ASME Winter Ann. Meeting, Miami Beach, FL, USA) 31, 67-74 (1985)

3. A.Yu. Kravtsova, D.M. Markovich, K.S. Pervunin, M.V. Timoshevskiy and K. Hanjalić, Int. J. Multiphase Flow 60, 119-134 (2014) 